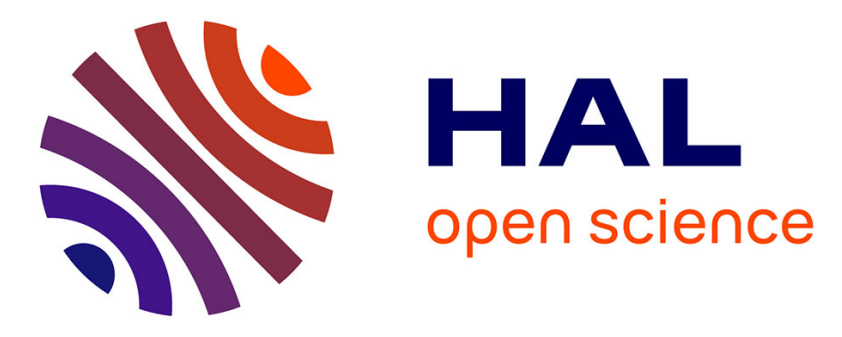

\title{
Wavenumber-Based Impedance Eduction with a Shear Grazing Flow
}

Rémi Roncen, Estelle Piot, Fabien Méry, Frank Simon, Michael G Jones, Douglas M Nark

\section{- To cite this version:}

Rémi Roncen, Estelle Piot, Fabien Méry, Frank Simon, Michael G Jones, et al.. Wavenumber-Based Impedance Eduction with a Shear Grazing Flow. AIAA Journal, 2020, pp.1-11. 10.2514/1.J059100 . hal-02859909

\section{HAL Id: hal-02859909 https://hal.science/hal-02859909}

Submitted on 8 Jun 2020

HAL is a multi-disciplinary open access archive for the deposit and dissemination of scientific research documents, whether they are published or not. The documents may come from teaching and research institutions in France or abroad, or from public or private research centers.
L'archive ouverte pluridisciplinaire HAL, est destinée au dépôt et à la diffusion de documents scientifiques de niveau recherche, publiés ou non, émanant des établissements d'enseignement et de recherche français ou étrangers, des laboratoires publics ou privés. 


\title{
Wavenumber-Based Impedance Eduction with a Shear Grazing Flow
}

\author{
R. Roncen*1, E. Piot ${ }^{1}$, F. Méry ${ }^{1}$, F. Simon ${ }^{1}$, M. G. Jones ${ }^{2}$, and D. M. Nark ${ }^{2}$ \\ ${ }^{1}$ ONERA /DMPE, Toulouse University, F-31055, Toulouse, France \\ ${ }^{2}$ NASA Langley Research Center, Hampton, VA 23681-2199, U.S.A.
}

\begin{abstract}
While intrinsic by definition, the impedance measured by impedance eduction has been shown to depend on the direction of the incident waves relative to the mean flow. The purpose of the present work is to evaluate whether part of the observed differences could stem from a biased wavenumber definition made during the impedance eduction process.

Comparisons are made between the results of impedance eductions with uniform flow and the Ingard-Myers boundary condition, with the 1D linearized Euler equations and with the 2D linearized Euler equations, i.e., in the cross section. Both numerical synthetic data and experimental data are used for the eduction of two sample liners, with bulk Mach numbers ranging from 0 to 0.3 , and at frequencies ranging from 400 to $3000 \mathrm{~Hz}$.

Results show that for a rectangular cross-section duct, the knowledge of the 2D flow profile in the cross section is valuable for impedance eduction. Using only $1 \mathrm{D}$ flow profiles bias the educed impedance estimation.
\end{abstract}

\section{Introduction}

Aircraft engines are one of the dominant noise sources for subsonic aircraft, resulting in a dense research field aimed at noise reduction inside the engine nacelle. In current turbofan engines, the inlet, bypass and exhaust parts of the nacelle are lined with acoustic liners. Liners consist essentially of a perforated plate backed by an air cavity, and have a resonator behavior.

The property employed to represent the locally reacting liner's acoustic behavior is the impedance $\zeta=R+\mathrm{j} \chi$ (with $R$ the resistance and $\chi$ the reactance), a complex frequency dependent quantity that relates the acoustic pressure to the normal acoustic particle velocity. The impedance is defined as an intrinsic parameter associated with a given liner configuration. However, estimates of the impedance value obtained from experimental measurements or direct numerical simulations depend on the sound pressure level [1-5] and the shear grazing flow velocity and profile [6-11], due to the appearance of nonlinear effects in the vicinity of the liner surface that are encompassed in the impedance modeling. The accurate knowledge of the influence that a complex flow has on the impedance is of prime importance when it comes to liner design and optimization [12, 13]. It

\footnotetext{
*Corresponding author: remi.roncen@onera.fr
} 
is also critical to be able to educe the impedance value of a liner subject to a shear grazing flow, which is known to complicate the measurement.

A wide number of different methods have been developed to educe the impedance, broadly separated into two different classes: straightforward and inverse methods.

$\triangleright$ Straightforward methods [14-16] use a measurement of the axial propagation wavenumber $k_{0,0}^{ \pm}$in conjunction with a model yielding a direct relationship between $k_{0,0}^{ \pm}$and $\zeta$. A uniform flow hypothesis is usually taken, and a declination of the classical Ingard-Myers impedance boundary condition [17, 18] is then used.

$\triangleright$ Indirect eduction methods [19]22] consist of measuring an acoustic field (pressure or velocity) and minimizing the difference between experimental and numerical fields obtained by solving partial differential equations (Convected Helmholtz Equations CHE or Linearized Euler Equations LEE) coupled with an impedance boundary condition.

While intrinsic by definition, the educed impedance of locally reacting materials has recently been shown to depend on the direction of the incident waves relative to the mean flow [23 26], i.e., upstream or downstream sources. This observed behavior has led to questions about the assumptions underlying the current eduction methods [27 29]: is the impedance definition insufficient, and should its modeling be augmented to take into account source direction? Or are the impedance eduction techniques inaccurate?

The purpose of the present study is to investigate the influence of the shear grazing flow profile on the impedance eduction process for upstream and downstream sources. To this end, a numerical synthetic experiment is first created in an attempt to reproduce the impedance mismatch observed in the NASA-GFIT test rig. In Sec. 2, acoustic data are generated by solving the LEE in the transverse section of a duct with a given "true" known impedance, while accounting for the presence of a shear grazing flow. In Sec. 3, a numerical eduction based on an updated eduction method is used with both source directions, mimicking the routinely conducted indirect eduction methods. In Sec. 4, an updated eduction version is then proposed and applied on experimental data retrieved on two different liner samples. A conclusion is then laid out in Sec. 5.

\section{Numerical synthetic experiment}

The goal of this section is to assess whether the impedance mismatch could arise from a biased definition of the wavenumber used in the straightforward method. To this end, a numerical synthetic experiment is set up, mimicking the NASA-GFIT aeroacoustic bench. The experimental setup configuration is first recalled, as well as the different methods available for wavenumber calculation.

\subsection{Experimental setup}

\subsubsection{Flow duct}

The GFIT duct was selected as the standard aeroacoustic bench to model numerically for the synthetic experiment due to its extensive use in impedance eduction studies [29, 30]. In this synthetic experiment, the lined section is assumed to be infinite, so that impedance discontinuities can be neglected. The GFIT has a cross-section geometry with a $50.8 \mathrm{~mm}$ width and $63.5 \mathrm{~mm}$ height, and typically operates over a frequency range of $400 \mathrm{~Hz}$ to $3000 \mathrm{~Hz}$, with Mach numbers 
reaching 0.5 at the centerline. Here, a case with a centerline Mach number of 0.3 is considered. A schematic of the numerical geometry used is given in Fig. 1 to introduce the axes notations in two different cases: the longitudinal case, and the transverse case. The flow goes from left to right ( $x$ direction). When the acoustic source is upstream of the lined section, the wave propagates in the same direction as the flow and the associated wavenumber is $k^{+}$. In the case of a downstream located source, the wave propagates against the flow and is associated with wavenumber $k^{-}$.

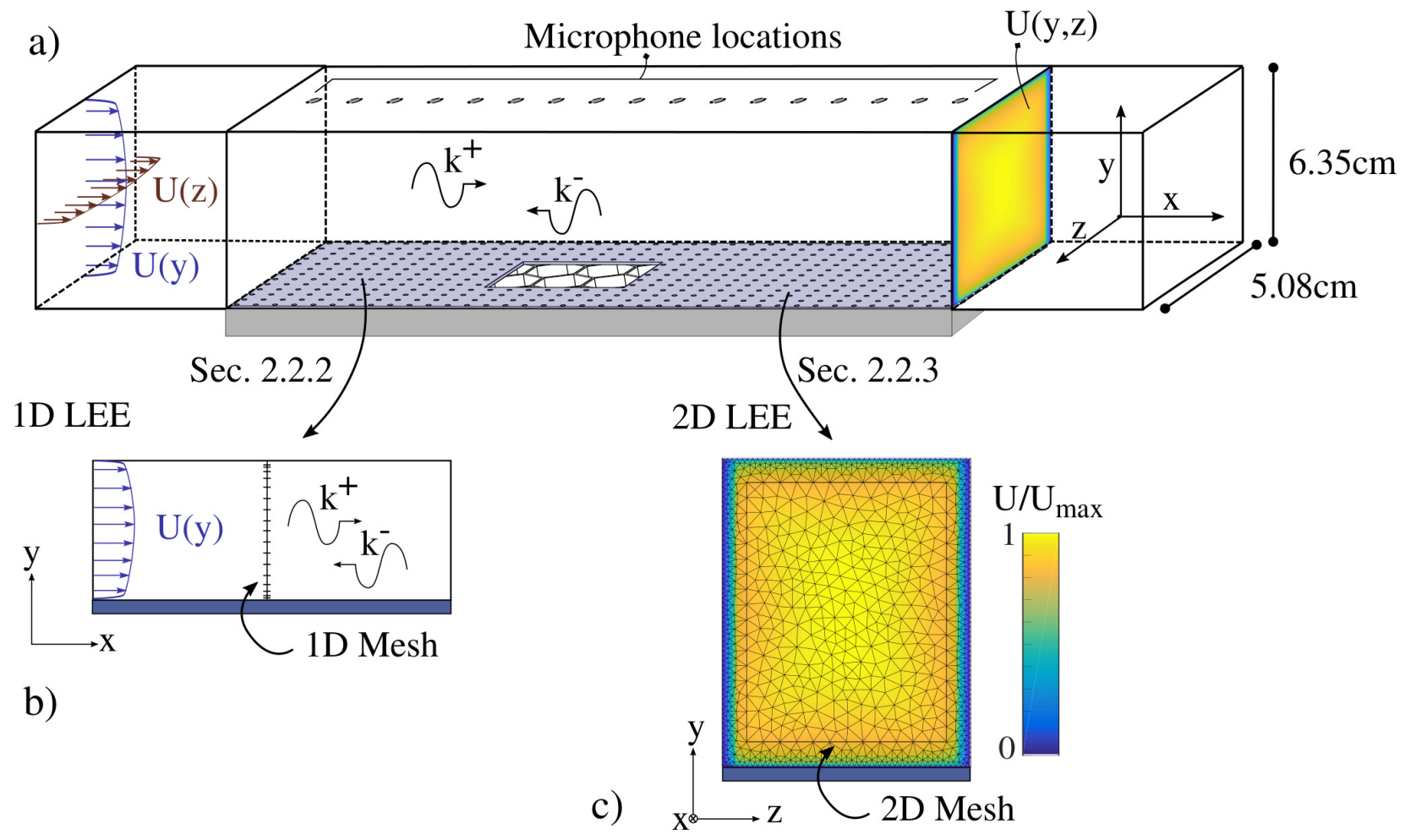

Figure 1: (a) Schematics of the GFIT (not to scale), with details of (b) the longitudinal case and (c) the transverse case.

\subsubsection{Liner configurations}

Two liners are considered in the current numerical synthetic study. The first liner (CT57) consists of ceramic tubes of diameter $0.635 \mathrm{~mm}$ and length $82.55 \mathrm{~mm}$. The surface porosity of the sample is $57 \%$. The CT57 has an impedance that is almost independent of the source SPL and of the grazing flow velocity [19]. The second liner (GE03) is a standard liner made of a perforated plate of thickness $t_{\mathrm{p} . \mathrm{p}}=0.635 \mathrm{~mm}$, perforation diameter $0.991 \mathrm{~mm}$ and porosity $15 \%$, backed by a honeycomb cavity of depth $38.1 \mathrm{~mm}$. The impedance of this liner only weakly depends on the source SPL and grazing flow velocity [19], due to its relatively high surface porosity. A sketch of the two test liners is represented in Fig. 2. The normalized numerical impedances $\zeta$ of both liners are given in Fig. 3, assuming that the influence of both the shear flow and the SPL nonlinearities is already taken into account. For the CT57, the impedance is taken as the one obtained without flow [31], assuming that the flow effect on the impedance is negligible. For the GE03, the impedance is taken as the average of the one educed in the presence of a grazing flow with a similar grazing flow profile [31], considering both upstream and downstream source locations. An interpolation is made on the full frequency range with a $25 \mathrm{~Hz}$ step. 


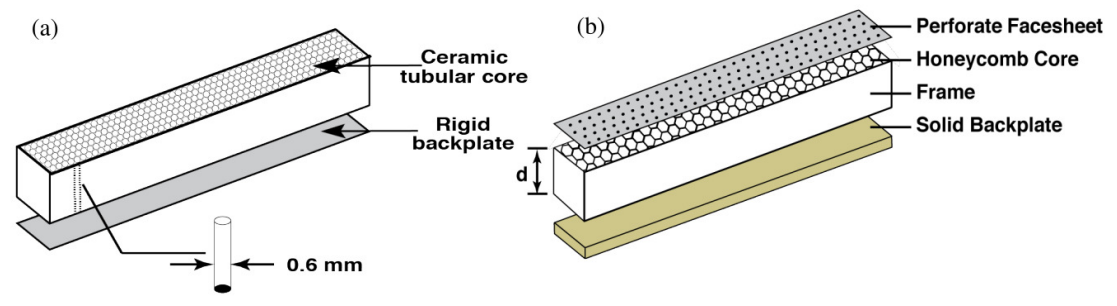

Figure 2: Sketches of (a) the CT57 and (b) the GE03 test liners (from Ref. [29]).

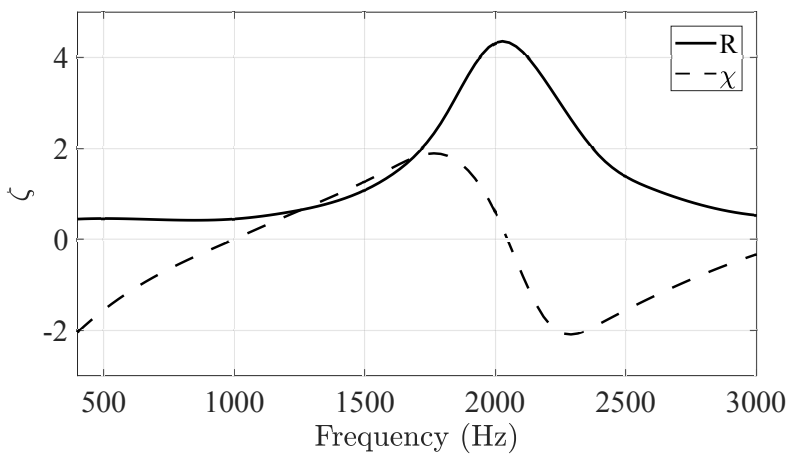

(a) CT57 numerical resistance (straight line) and reactance (dashed line).

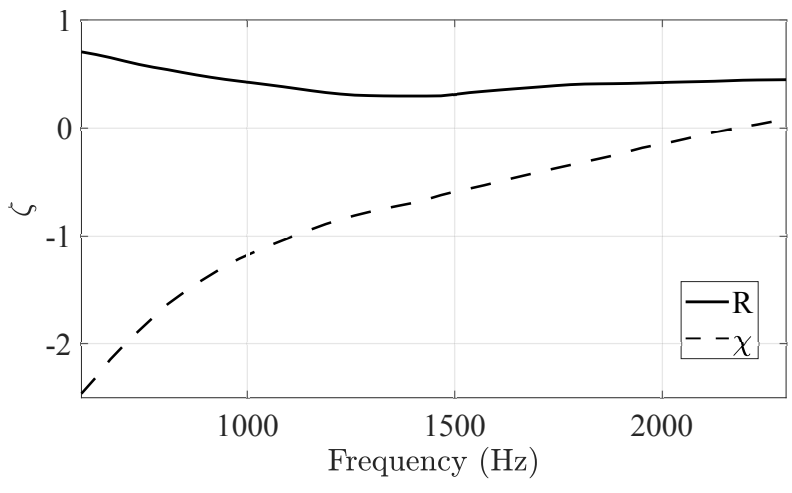

(b) GE03 numerical resistance (straight line) and reactance (dashed line).

Figure 3: Impedance values of the synthetically generated liner samples.

The influence of the source location on the educed impedance of both liners was shown experimentally in prior studies, using indirect eduction methods [29, 31].

\subsection{Direct problem: calculation of the wavenumber}

Using the Kumaresan and Tufts [32] approach, it is possible to experimentally estimate the axial wavenumbers of the modes propagating above the lined duct section [14], using only pressure measurements at the wall opposite the liner (the GFIT lined section is equipped with 23 microphones). At the frequencies of interest, only the plane wave mode propagates. A wavenumber-based eduction process consists in comparing the experimental measurement of the wavenumber to a model relating the impedance $\zeta^{ \pm}$( + for upstream source, - for downstream source) to the measured wavenumber $k_{x}^{ \pm}$as

$$
\zeta^{ \pm}=G^{-1}\left(k_{x}^{ \pm}\right)
$$

with $G$ a direct operator mapping the impedance space onto the wavenumber space. Three of these models are presented hereafter, by increasing order of complexity.

\subsubsection{Uniform mean flow hypothesis}

When a uniform flow hypothesis is made (shear layer is assumed thin), an explicit relationship (subscript 0D) exists between the impedance and the wavenumber, as given in Ref. [23]:

$$
\zeta_{0 \mathrm{D}}^{ \pm}=G_{0 \mathrm{D}}^{-1}\left(k_{x}^{ \pm}\right)=\frac{\left(\mathrm{j} \omega-\mathrm{j} M_{0} k_{x}^{ \pm}\right)^{2}}{\mathrm{j} \omega k_{1} \tan \left(k_{1}\right)},
$$


where $M_{0}$ is the bulk Mach number and $k_{1}=\left(\omega-M_{0} k_{x}^{ \pm}\right)-\left(k_{x}^{ \pm}\right)^{2}$, and $\omega$ is the angular frequency of the plane wave. This mapping, relying on the Ingard-Myers boundary condition [17, 18], is the basis of the straightforward methods used in the literature.

\subsubsection{One dimensional LEE}

To avoid the hypothesis of a uniform mean flow, the Linearized Euler Equations (LEE) can be used. For an inviscid homentropic perfect gas flow inside the duct, the Euler equations are

$$
\begin{gathered}
\frac{\partial \rho}{\partial t}+\boldsymbol{\nabla} \cdot(\rho \boldsymbol{V})=0, \\
\rho \frac{\mathrm{D} \boldsymbol{V}}{\mathrm{D} t}+\nabla p=0,
\end{gathered}
$$

where $p$ is the pressure, $\rho$ the density, and $\boldsymbol{V}$ is the velocity with components $U, V$ and $W$ in the $x, y, z$ directions, respectively (see Fig. 1). The convective derivation is written $\mathrm{D} / \mathrm{D} t=$ $\partial / \partial t+(\boldsymbol{V} \cdot \boldsymbol{\nabla})$. The flow field is decomposed into a static field (subscript 0) and a small-amplitude unsteady perturbation (tilde symbol) as

$$
(\boldsymbol{V}, p)=\left(\boldsymbol{V}_{0}, p_{0}\right)+(\tilde{\boldsymbol{V}}, \tilde{p}),
$$

with $\gamma=c_{p} / c_{v}$ the gas adiabatic index. Linearizing Eqs. 3 with respect to the small perturbations lead to the LEE,

$$
\begin{aligned}
\partial_{t} \boldsymbol{\varphi}+\boldsymbol{A}_{x} \partial_{x} \boldsymbol{\varphi}+\boldsymbol{A}_{y} \partial_{y} \boldsymbol{\varphi}+\boldsymbol{A}_{z} \partial_{z} \boldsymbol{\varphi}+\boldsymbol{B} \boldsymbol{\varphi} & =0, \quad \text { in } \Omega, \\
\tilde{p} & =\tilde{\boldsymbol{u}} \cdot \boldsymbol{n}, \quad \text { on } \Gamma_{\zeta}
\end{aligned}
$$

with $\boldsymbol{\varphi}=(\tilde{\boldsymbol{u}}, \tilde{p})$, and with the $\boldsymbol{A}_{j}$ and $\boldsymbol{B}$ matrices depending on the steady flow fields [21, 33]. The physical domain is denoted $\Omega$, while the impedance boundary condition (Eq. 6) is applied on $\Gamma_{\zeta}$, whose inward normal is $\boldsymbol{n}$.

In the case where the geometry is considered one-dimensional (hereafter 1D), the wave propagates in the $x$ direction over an infinite lined section with the shear flow only considered in the vertical $y$ direction. The problem is then invariant in the transverse $z$ direction and the $\partial_{z}$ term can be dropped in Eq. 5. Due to the stationarity of the base flow and the $x$ invariance of the problem, the acoustic perturbation is taken under the form

$$
\boldsymbol{\varphi}=\boldsymbol{\varphi}(y) e^{-\mathrm{j} k_{x} x+\mathrm{j} \omega t}
$$

The harmonic LEE can be written in the form of a generalized eigenvalue problem as

$$
\left(\mathrm{j} \omega+\boldsymbol{A}_{y} \partial_{y}+\boldsymbol{B}\right) \boldsymbol{\varphi}=\mathrm{j} k_{x} \boldsymbol{A}_{x} \boldsymbol{\varphi}
$$

which is rewritten for convenience as

$$
\boldsymbol{D} \varphi=k_{x} \boldsymbol{E} \boldsymbol{\varphi}
$$

A number of ways are available to solve this eigenvalue problem, all requiring the discretization of the $\partial_{y}$ operator in Eq. 8 (the impedance boundary condition, Eq. 6, is strongly enforced by replacing the continuity equation in the general matrix formulation). In the present work, the strategy of Ref. [34] is followed, where the LEE are projected onto a Chebyshev polynomials basis 
to discretize the problem. The whole procedure of creating the eigenvalue problem and solving it is written as

$$
G_{1 \mathrm{D}}\left(\zeta_{1 \mathrm{D}}^{ \pm}\right)=k_{x}^{ \pm}
$$

The educed impedance can then be obtained through

$$
\zeta_{1 \mathrm{D}}^{ \pm}=G_{1 \mathrm{D}}^{-1}\left(k_{x}^{ \pm}\right)
$$

which represents the resolution of an inverse problem.

\subsubsection{Two dimensional LEE}

When the problem is not assumed invariant in the transverse $z$ direction, the $\partial_{z}$ term cannot be neglected in Eq. 5. The stationarity of the base flow and the harmonic wave hypothesis lead to an acoustic perturbation of the form

$$
\boldsymbol{\varphi}=\boldsymbol{\varphi}(y, z) e^{-\mathrm{j} k_{x} x+\mathrm{j} \omega t}
$$

The LEE can once again be written under the form of a generalized eigenproblem as

$$
\left(\mathrm{j} \omega+\boldsymbol{A}_{y} \partial_{y}+\boldsymbol{A}_{z} \partial_{z}+\boldsymbol{B}\right) \boldsymbol{\varphi}=\mathrm{j} k_{x} \boldsymbol{A}_{x} \boldsymbol{\varphi}
$$

which is written for convenience as

$$
D^{\prime} \varphi=k_{x} \boldsymbol{E}^{\prime} \varphi
$$

The strategy of Ref. [33] is followed for the resolution of the eigenproblem, using a Discontinuous Galerkin scheme for the discretization of the $\partial_{y}$ and $\partial_{z}$ operators (the impedance boundary condition is weakly enforced via a flux matrix). A shift invert Arnoldi method is then used to retrieve the eigenvalues of interest, due to the singular nature of the eigenproblem. To initialize the shift in Arnoldi's method, the initial eigenvalue is taken as the wavenumber calculated using the uniform flow hypothesis: $k_{\text {init }}=G_{0}(\zeta)$. The whole procedure of creating the eigenvalue problem and solving it is written as

$$
G_{2 \mathrm{D}}\left(\zeta_{2 \mathrm{D}}^{ \pm}\right)=k_{x}^{ \pm}
$$

The educed impedance can then be obtained through

$$
\zeta_{2 \mathrm{D}}^{ \pm}=G_{2 \mathrm{D}}^{-1}\left(k_{x}^{ \pm}\right)
$$

which represents the resolution of an inverse problem.

\subsubsection{Comparison between wavenumber calculations}

The calculation of the wavenumber is now performed for the two considered liners, using the three different numerical methods presented herein. The impedance $\zeta$ is fixed at the value in Fig. 3. Then, the wavenumbers $k_{x}^{ \pm}$are calculated following Eq. 2, 10 or 15 . Two different flows are considered, as depicted in Fig. 4, to evaluate the influence of the shear flow profile on the calculated wavenumber. In addition, both the uniform flow method (0D) and the 1D LEE method are used twice for each flow. This is done in order to evaluate the influence of the Mach number definition on the calculated wavenumber. In the first scenario, one uses the midspan velocity profile as the base flow profile, while in the second scenario (" $M_{b}$ corr" entries in the legend) this profile 
is corrected to obtain a $1 \mathrm{D}$ flow profile whose average bulk value is the average value of the $2 \mathrm{D}$ flow profile, which is lower:

$$
\int M_{b}(y, z) \mathrm{d} y \mathrm{~d} z<\int M_{b}\left(y, z=z_{\max } / 2\right) \mathrm{d} y .
$$

Flow 1 and 2 are derived from the same hyperbolic velocity profile given in Ref. [35, Eq. 4]:

$$
M_{0}(r)=M_{c} \tanh \left(\frac{1-|r|}{\delta}\right)+M_{c}\left[1-\tanh \left(\frac{1}{\delta}\right)\right]\left[\frac{1+\tanh \left(\frac{1}{\delta}\right)}{\delta}+1+|r|\right](1-|r|)
$$

with $r \in[-1,1]$ and $M_{c}$ the centerline Mach number. The nondimensional boundary layer thickness $\delta \in(0,1]$ is set to 0.1 for flow 1 , and to 0.5 for flow 2 . In practice, a mapping is performed to have $r$ in a suitable interval, a tensorization is performed to obtain a 2D flow on $(y, z)$, and a normalization is applied to obtain the required bulk Mach number.

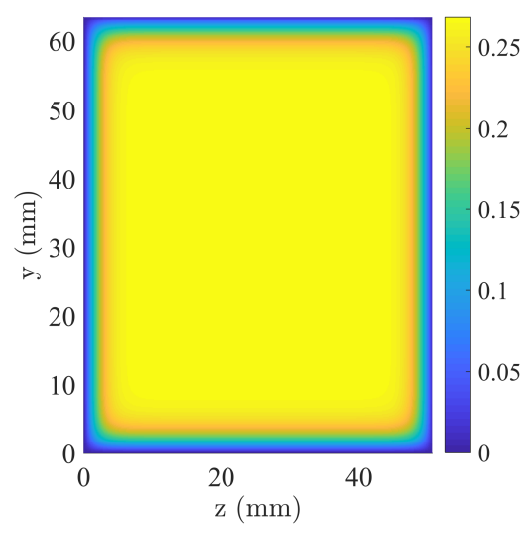

(a) Flow 1.

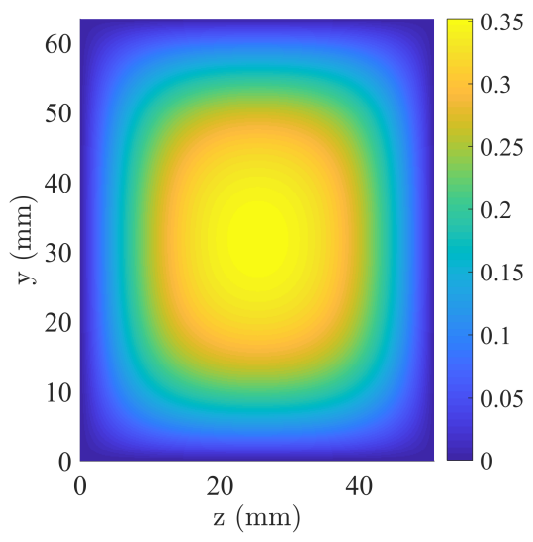

(b) Flow 2.

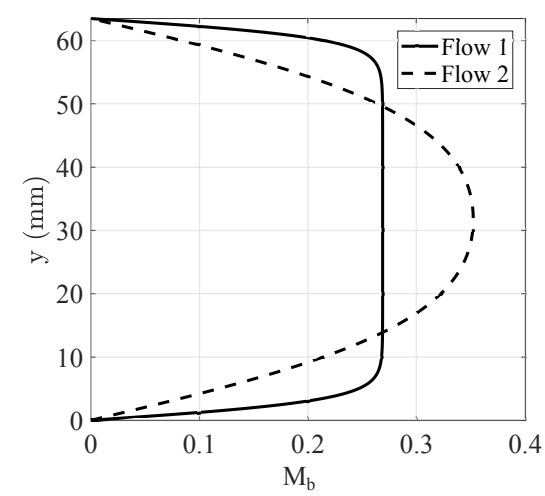

(c) Mach number profile at midspan location.

Figure 4: Flow profiles used for the wavenumber calculation.

The obtained wavenumbers are given in Fig. 5 for the CT57 (conclusions being similar for the GE03). For clarity, the results are constrained to frequencies of $400-1800 \mathrm{~Hz}$, as the more interesting features occur within this frequency range.

The following conclusions can be drawn regarding the definition of the wavenumber:

$\triangleright$ A clear mismatch is observed between the different wavenumber values. When the flow profile is strongly sheared (flow 1), the mismatch is lower between 1D and 2D wavenumbers (black and blue curves), as compared to the case of flow 2, which has a Poiseuille-like flow profile.

$\triangleright$ The mismatch between the different wavenumber values is higher for $k_{x}^{-}$, i.e., for the waves propagating against the flow.

$\triangleright$ There exist frequencies at which the wavenumber mismatch is almost null for all the models.

$\triangleright$ While updating the flow profile to account for the true average bulk Mach number seems to correct some of the observed mismatch (dashed curves appear closer to the reference 2D model), it is not sufficient. 


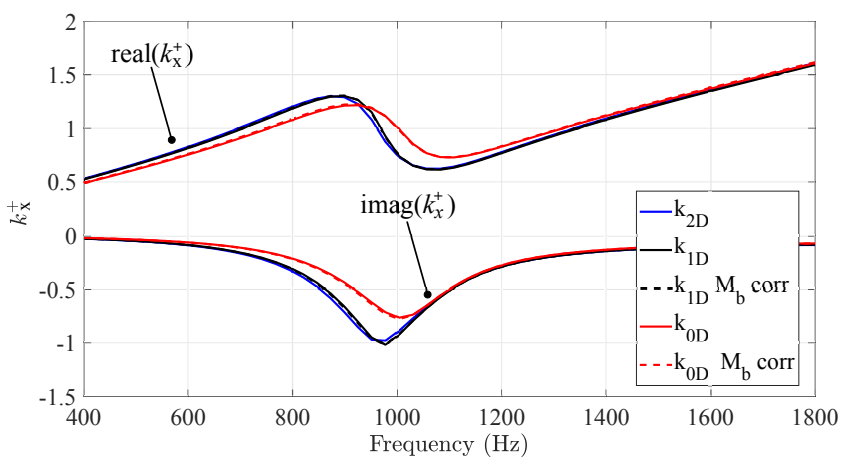

(a) Flow 1: $k_{x}^{+}$(upstream source).

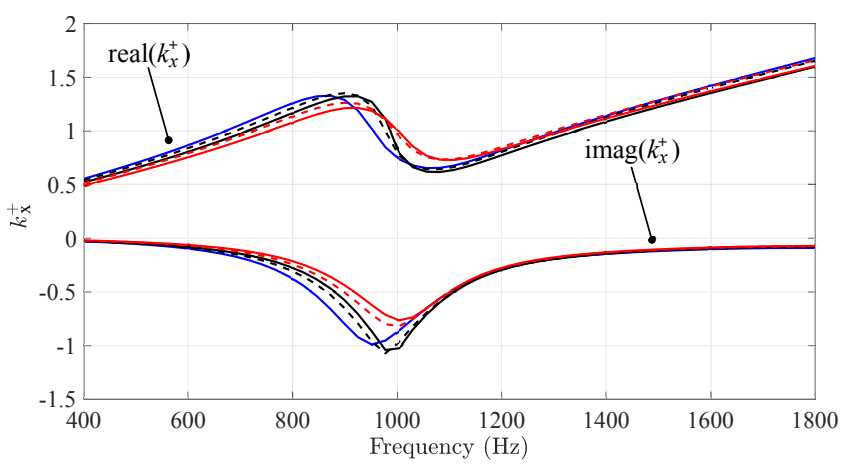

(c) Flow 2: $k_{x}^{+}$(upstream source).

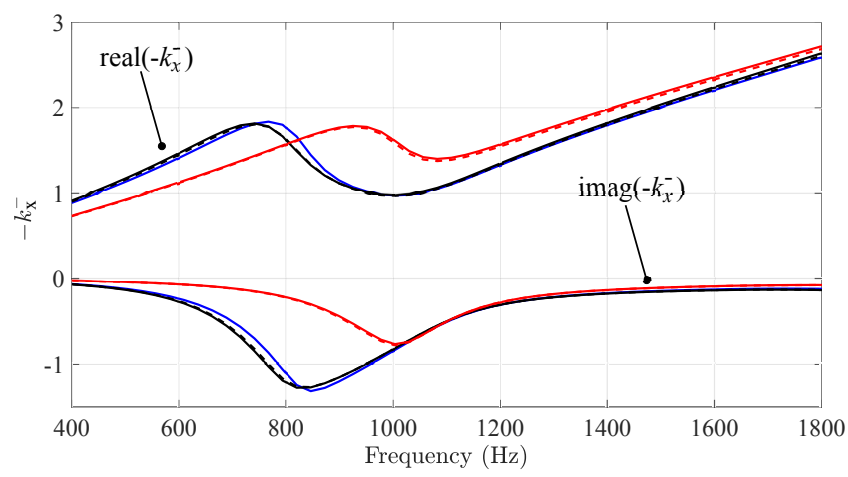

(b) Flow 1: $-k_{x}^{-}$(downstream source).

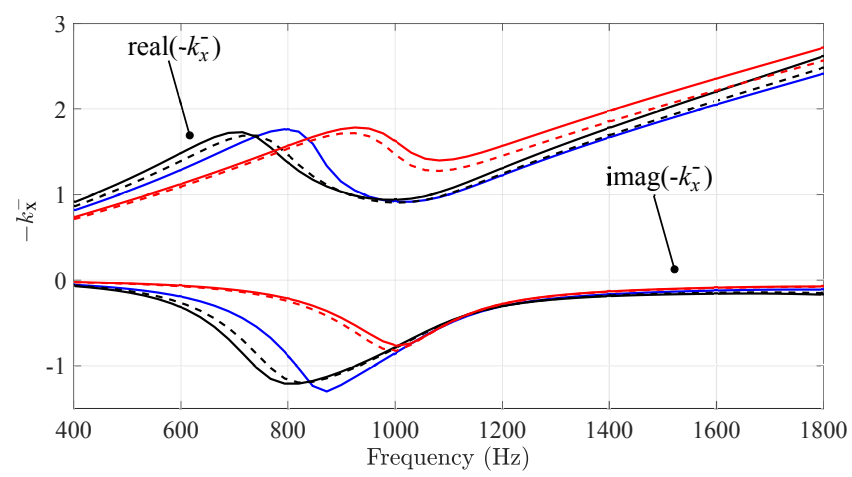

(d) Flow 2: $-k_{x}^{-}$(downstream source).

Figure 5: Plane wave wavenumbers obtained from the CT57 impedance and for the flow profiles of Fig. 4

It thus stands to reason that the eduction mismatch problem observed in the literature could be caused by a bias in the wavenumber definition, since the transverse shear is usually not taken into account. For methods relying on a direct measurement of the wavenumber, a work-around could consist in performing the eduction with the 2D LEE transverse model to compute the wavenumbers, as considered in Sec. 3. For indirect eduction methods, it is not as simple, since the pressure measurements are performed above lined as well as rigid wall sections, where the wavenumber mismatch might be different. In addition, the impedance discontinuity at the linerwall junction can create multiple evanescent (cut off) modes [33], which also have a mismatch different than the one with the plane wave mode. Note that while correcting the Mach number might allow for a lower wavenumber mismatch at certain frequencies, it cannot fully bridge the gap. This is the reason why such corrections were only partially successful in reducing the impedance eduction mismatch [29] in earlier attempts.

To check our claim that the wavenumber definition is a potential error source resulting in an impedance eduction mismatch, a numerical eduction is first performed on the synthetic data shown in the present section, setting the 2D LEE transverse model as the reference measurement, and using the 1D LEE model to try and educe the impedance value in both directions. 


\section{Numerical Eduction}

This section presents the eduction problem used on the numerically generated synthetic data, to reproduce the eduction performed in practice and observe the influence of the wavenumber definition on the educed impedance value. First, the eduction strategy is presented.

\subsection{Eduction strategy}

The 2D transverse LEE are solved in the cross-section as the eigenvalue problem (Eq. 15) in order to obtain the propagating mode wavenumbers of interest $k_{2 \mathrm{D}}^{+}$and $k_{2 \mathrm{D}}^{-}$in the presence of a (numerical) shear grazing flow. The reference impedance values displayed in Fig. 3 are imposed at the wall, in order to mimic an experiment in the GFIT. The shear grazing flow is taken as the one experimentally measured in the GFIT cross-section (average of measurements conducted upstream and downstream of the liner), that was used to create Fig. 4a. These wavenumbers are considered as the reference wavenumbers, which will be used for the eduction. The next step consists in solving the inverse problem (Eq. 11), thus finding an impedance value that minimizes the mismatch between $k_{2 \mathrm{D}}^{ \pm}$and a model as

$$
\zeta^{ \pm}=\underset{Z}{\operatorname{argmin}}\left(\left\|G_{1 \mathrm{D}}(Z)-k_{2 \mathrm{D}}^{ \pm}\right\|_{2}\right)
$$

in the case where the model is taken as the one in Sec. 2.2.2. Alternatively, one could use the model based on the uniform flow hypothesis (which would result in a method similar to the classical straightforward approach), or the 2D LEE model. Hereafter, the identification is performed using only the 1D LEE model, since it appeared to be the closest to the reference measurement in Sec. 2.2.4. No Mach correction is applied onto the 1D flow profile, which is taken as the midspan profile, as usually done in practice [19, 21, 23]. The eduction is performed separately on $k_{2 \mathrm{D}}^{+}$and $k_{2 \mathrm{D}}^{-}$.

\subsection{Results}

Eduction results are displayed in Fig. 6 for the CT57 and in Fig. 7 for the GE03. In both cases, the eduction obtained using a classical indirect method (see Ref. [31]) on experiments performed in GFIT with these liners are given as well.

While the reference impedance (red curve) was taken arbitrarily for each material, it appears that the educed impedance reproduces, at least qualitatively, the impedance mismatch observed with the indirect method applied to true experimental data.

Looking at the resistance of the CT57 in Fig. 6, near the antiresonance $(\approx 2000 \mathrm{~Hz})$, a very similar "shift" in frequency is observed: the upstream source experimental eduction and the numerical eduction based on $k^{+}$both predict an antiresonance at a lower frequency, while the opposite is true for the downstream experimental eduction and $k^{-}$-based numerical eduction. The difference in frequency seems qualitatively the same in both the numerical and experimental case, but the frequency resolution of the experimental eduction is not fine enough to obtain a precise value for comparison.

In addition, the maximum of resistance is different at the antiresonance, with a slight overestimation for the $k^{+}$-based eduction, and an underestimation for the $k^{-}$-based eduction. While the "true" experimental impedance value is unknown in this case, the discrepancy in maximum 


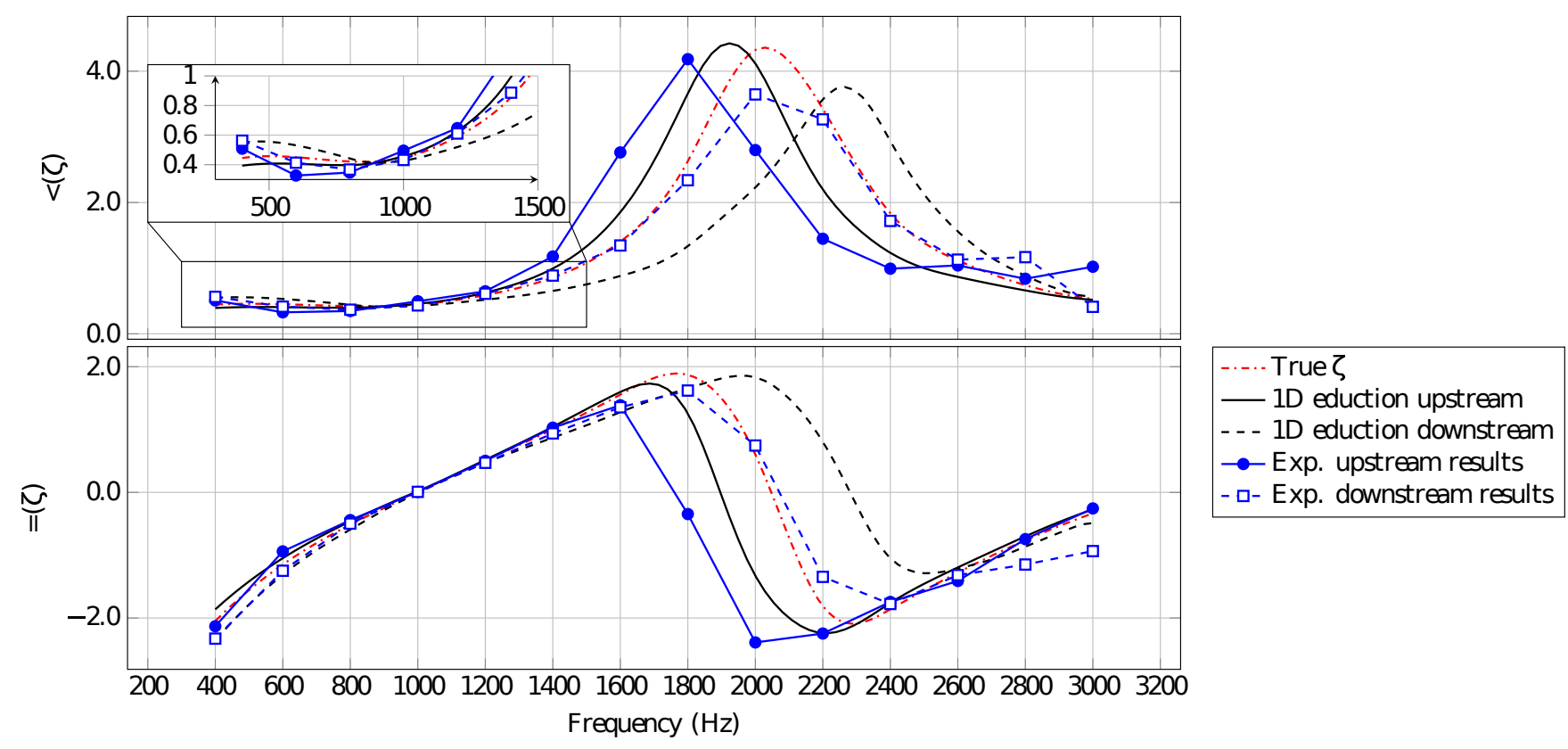

Figure 6: CT57 educed impedance, numerical case (black lines), and experimental results from Ref. [31] (symbols).

resistance value is still observed at antiresonance, with a difference that is quantitatively similar to the one observed during the numerical eductions.

Another interesting similarity between the CT57 experimental and numerical eductions occurs at lower frequencies, near the resonance. Looking at the zoomed frequency window in Fig. 6, it is observed that the mismatch of both the experimental and numerical educed resistance $\Re\left(\zeta^{+}-\zeta^{-}\right)$ has a sign change near $\approx 900 \mathrm{~Hz}$. We note that in Ref. [29, Fig. 8], a large uncertainty in the educed axial wavenumber was found at $800 \mathrm{~Hz}$ for the CT57.

As for the GE03 liner in Fig. 7, a correct qualitative agreement is obtained between experimental and numerical eductions (recall that the true experimental impedance is unknown). It is observed that the resistance mismatch sign shift $\left(\operatorname{sgn}\left(\Re\left(\zeta^{+}-\zeta^{-}\right)\right)\right)$still occurs in both cases, albeit at different frequencies (see the two magnifying windows in Fig. 7).

Partial conclusion Using a 1D propagation model for a 2D configuration results in an apparent impedance mismatch, which is significant at certain frequencies. This is true even as the error on the wavenumber in Fig. 5 is not always important.

\section{Experimental validation}

This section presents an updated eduction strategy applied to experimental data obtained in the GFIT for two liner samples, the CT57 and the GE03, in the presence of a shear grazing flow of $M_{b} \approx 0.25$.

\subsection{Updated eduction strategy}

Now that the wavenumber definition has been shown to generate an eduction impedance bias, an updated inference strategy is proposed. The experimentally measured wavenumber (using 


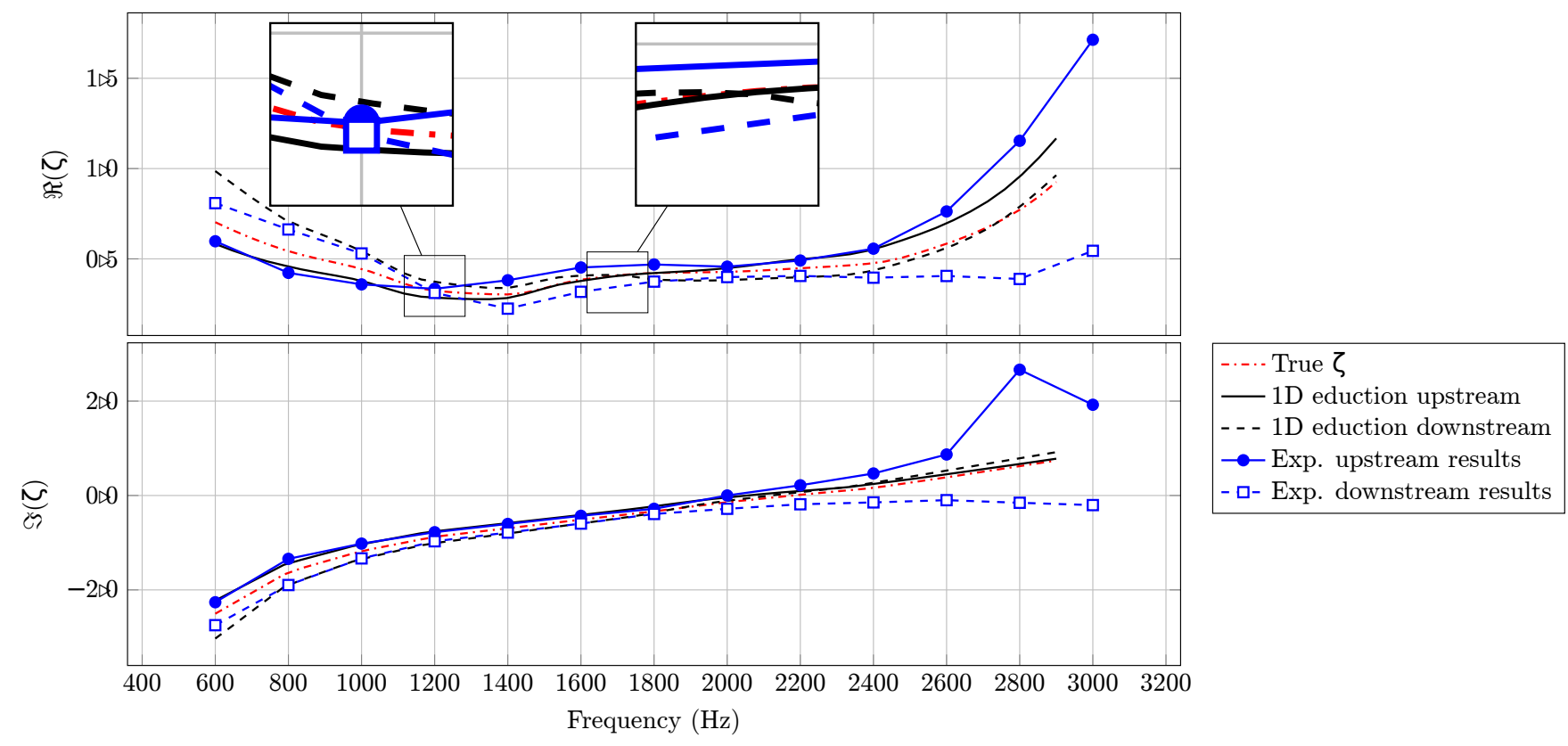

Figure 7: GE03 educed impedance, numerical case (straight lines), and experimental results from Ref. [31] (symbols).

Kumaresan and Tufts [32] approach) is now compared to the 2D LEE model iteratively, until a minimum is reached:

$$
\zeta^{ \pm}=\underset{Z}{\operatorname{argmin}}\left(\left\|G_{2 \mathrm{D}}(Z)-k_{\exp }^{ \pm}\right\|_{2}\right) .
$$

The goal of this approach is to get rid of the bias introduced by a wrong definition of the wavenumber in 3D aeroacoustic benches such as the GFIT. A schematic of the new approach is given in Fig. 8 .

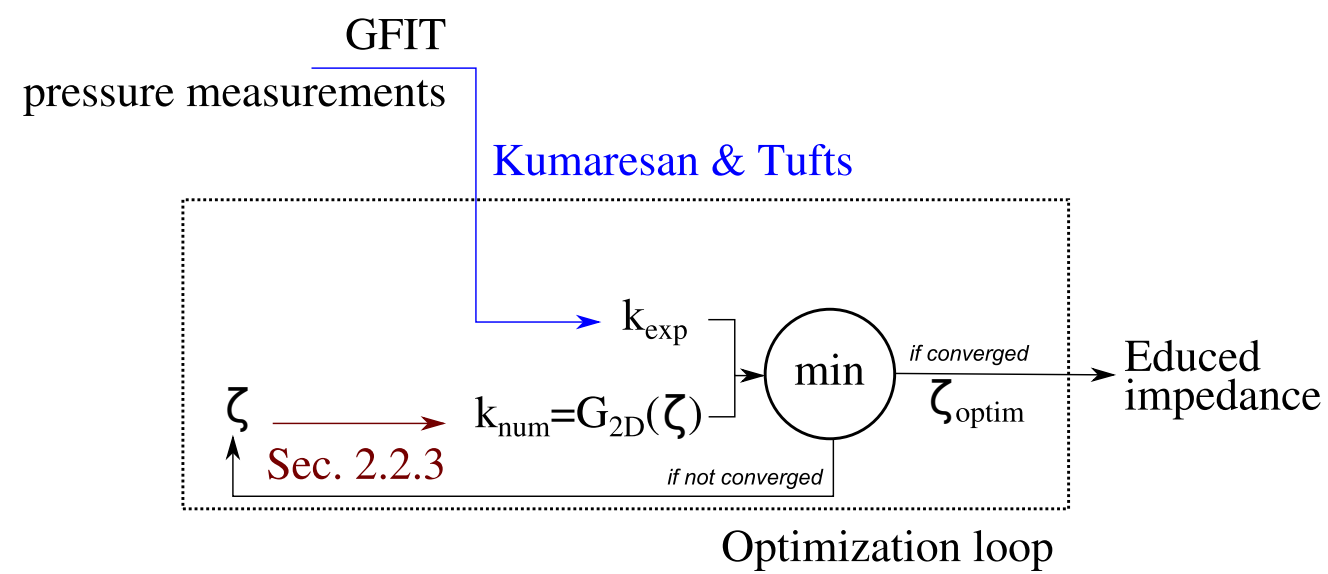

Figure 8: Schematics of the eduction method.

Additionally, an objective Bayesian inference [36, Chap. 8] can be performed in order to quantify the uncertainties on the educed impedance, given uncertain measurements of $k_{\exp }^{ \pm}$(see Ref. [22, 37] for an application to eduction using pressure measurements or laser doppler velocimetry). Bayesian inference can be seen as a rule to update one's information on a quantity, given a new observation. 
The goal becomes that of finding $\pi\left(\zeta^{ \pm} \mid k_{\exp }^{ \pm}\right)$, i.e., the probability density function of the impedance, updated by the knowledge contained in a new observation (here, $\left.k_{\exp }^{ \pm}\right)$. In this formalism, both the impedance $\zeta^{ \pm}$and the observation $k_{\exp }^{ \pm}$are considered as realizations of random variables having a normal distribution. The impedance $\zeta^{ \pm}$is separated into its resistance and reactance, both considered as random variables with a normal distribution of variance $\sigma_{\Re(\zeta)}^{2}$ and $\sigma_{\Im(\zeta)}^{2}$, respectively. A number of $n=10$ measurements, assumed uncorrelated, $k_{i}^{ \pm}$are performed. To achieve uncorrelated measurements, the measurement campaign was carried over the course of a month. Each sample was physically removed and reinstalled every six tests (two at $M_{b}=0$, two at $M_{b} \approx 0.25$, and two at $M_{b} \approx 0.42$ not used in this paper). Between each test, the test conditions (Mach number) were forced to change in the following pattern: $(0-0.3-0.5-0.3-0.5-0)$. The two samples were interchanged after the six measurements, so that the full set of tests for either sample would be spread over as long a time as possible. The procedure was performed 5 times, resulting in the 10 measurements used in this work.

Using Bayes' theorem, one has

$$
\pi\left(\zeta^{ \pm} \mid k_{\exp }^{ \pm}\right) \propto \pi\left(k_{\exp }^{ \pm} \mid \zeta^{ \pm}\right) \cdot \pi\left(\zeta^{ \pm}\right)
$$

where $\pi\left(\zeta^{ \pm}\right)$is the prior density, representing already existing knowledge one has on the impedance, and $\pi\left(k_{\exp }^{ \pm} \mid \zeta^{ \pm}\right)$is the likelihood, representing the new information contained in the experiment (i.e., it represents the distance between a numerical model output and an experiment, in terms of probabilities). In this work, an objective Bayesian inference is attempted, meaning that the prior density $\pi\left(\zeta^{ \pm}\right)$is taken as a uniform density of large support, in order to translate our lack of prior information on the impedance.

With the Gaussian hypothesis used in this work and the uniform prior, classical estimators (mean, maximum likelihood, maximum a posteriori) are equivalent. Here we choose the mean, denoted by a bar as $\boldsymbol{\bullet}$.

The impedance mean is found by maximizing the likelihood

$$
\bar{\zeta}^{ \pm}=\underset{Z}{\operatorname{argmax}}\left(\pi\left(k_{\exp } \mid Z\right)=\frac{1}{\left(2 \pi \sigma^{2}\right)^{n / 2}} \prod_{i=1}^{n} e^{-\frac{\left|G_{2 \mathrm{D}}(Z)-k_{\mathrm{i}}^{ \pm}\right|}{2 \sigma}}\right),
$$

where $\sigma^{2}$ is the observation and model error variance. Note that when no prior information is available, the above problem is equivalent to the one of Eq. 19. The variance of $\zeta^{ \pm}$is then found by calculating the covariance matrix of $\pi\left(\zeta^{ \pm} \mid k_{\exp }^{ \pm}\right)$, which is done by evaluating the Hessian matrix (by finite difference) of $\pi\left(k_{\exp } \mid Z\right)$ at $Z=\bar{\zeta}^{ \pm}$. The knowledge of $\sigma_{\Re(\zeta)}^{2}$ and $\sigma_{\Im(\zeta)}^{2}$ allows the display of credibility intervals, thus quantifying the relative influence of uncertainties on the impedance eduction results.

\subsection{Results}

The experimentally measured wave numbers are shown as a function of frequency for the CT57 and GE03 materials in Figs. 9-10. Their associated 95\% confidence intervals correspond to two standard deviations calculated with the 10 measurements that were performed. In some cases, the wavenumber extraction strategy of Kumaresan and Tuft 32 failed to find a physically coherent wavenumber value, and these points were removed from the graphs. The standard deviation appears quite limited compared to the large range of possible wavenumber values.

Eduction results are displayed in Figs. (11, 12, where the new eduction strategy is compared to the classical straightforward method, used with a mean flow value taken as the one obtained 


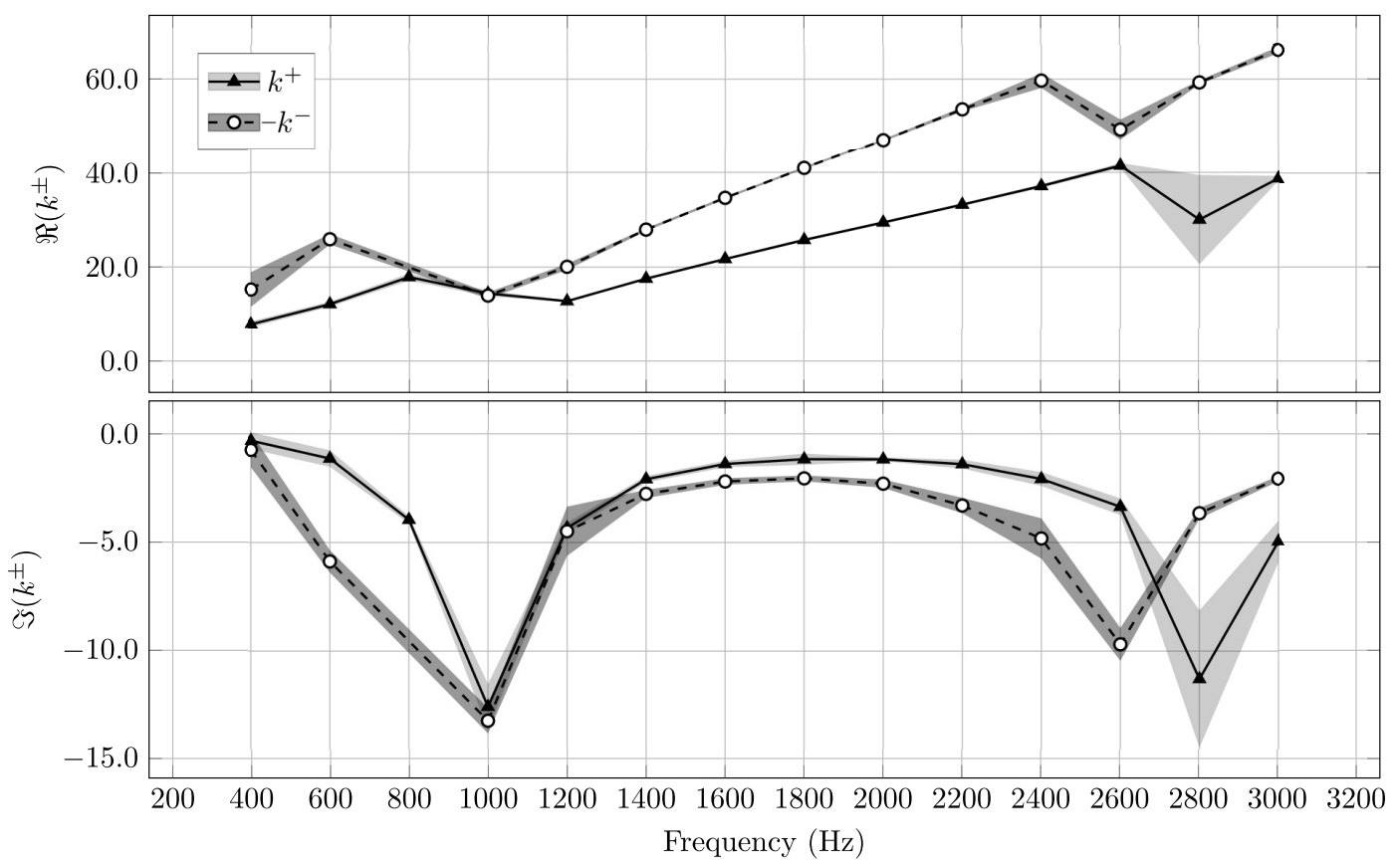

Figure 9: Experimental axial wavenumber $k^{+}$and $-k^{-}$of the CT57.

when considering only the midspan flow profile. 95\% credibility intervals (CI) are displayed for the $2 \mathrm{D}$ eduction results, meaning that the true value of $\zeta$ has a $95 \%$ chance of being within the intervals, given the present set of measurements and model used.

For the CT57 in Fig. 11, some important features are listed below.

$\triangleright$ Compared to the straightforward (0D) eduction approach, the impedance mismatch obtained with the updated $(2 \mathrm{D})$ strategy is reduced near the antiresonance $(\approx 2000 \mathrm{~Hz})$. The antiresonance position is not shifted anymore when considering upstream and downstream configurations. A similar result was obtained in Ref. [29, Fig. 6] by slightly adjusting the Mach number in the 0D method.

$\triangleright$ For frequencies above the antiresonance, the mismatch behavior is different for the two eductions, when considering the reactance $\chi$ : the upstream educed reactance is higher than the downstream educed one for the straightforward eduction, while the opposite is true for the updated 2D-LEE eduction.

$\triangleright$ At frequencies below $1500 \mathrm{~Hz}$, the resistance mismatch is lower for the straightforward method, compared to the updated 2D-LEE eduction, with almost no mismatch.

$\triangleright$ For the reactance, the mismatch seems increasingly high as the frequency decreases below the resonance frequency. However, the mismatch "sign" is once again opposed for both eductions. Note that the observed mismatch for the reactance at low frequency using the straightforward method is comparable to the one obtained independently in Ref. [23, Fig. 4].

$\triangleright$ The uncertainty on the educed impedance seems to correlate with the impedance mismatch. At low frequencies and near the antiresonance, the uncertainty is increased because the liner creates almost no acoustic damping. 


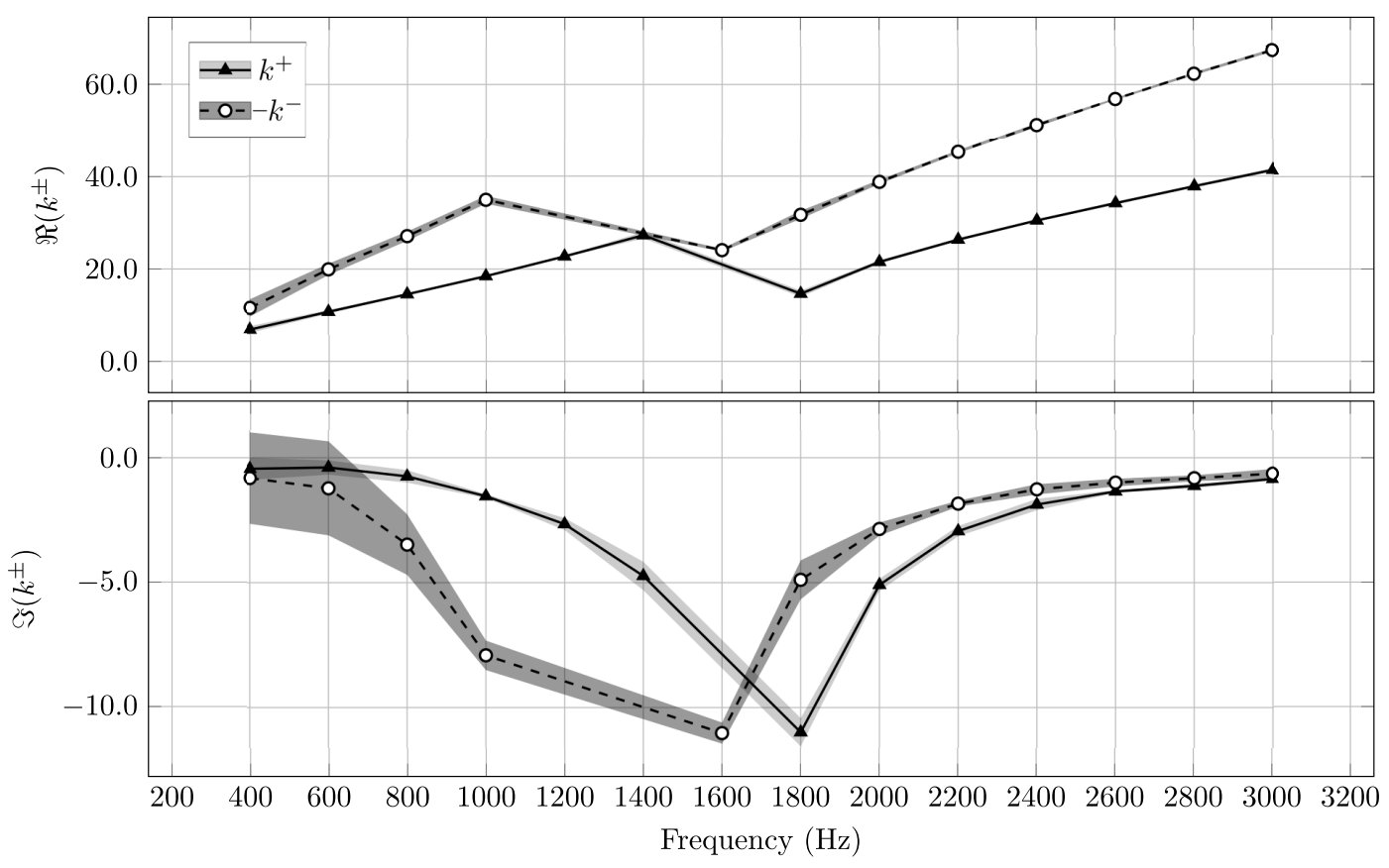

Figure 10: Experimental axial wavenumber $k^{+}$and $-k^{-}$of the GE03.

$\triangleright$ The uncertainties associated with the downstream eduction are always smaller than the upstream eduction. This observation has already been made in Ref. [31] when considering a classical approach to eduction. This discrepancy is due to the $k^{-}$wavenumber being more sensitive to a change in impedance compared to the $k^{+}$wavenumber.

For the GE03 in Fig. 12 , observations are listed below.

$\triangleright$ At low frequencies, the mismatch is quantitatively similar for both methods. At high frequencies, the $2 \mathrm{D}$ eduction cancels most of the impedance mismatch. A very similar result was obtained by slightly adjusting the Mach number in Ref. [29, Fig. 7]

$\triangleright$ The uncertainties on the impedance educed by the 2D eduction method seem to strongly correlate with the impedance mismatch amplitude observed with the 0D eduction approach. Just below the resonance frequency $(\approx 1800 \mathrm{~Hz})$, both the impedance mismatch and uncertainties are minimal.

$\triangleright$ In the case of the 2D eduction approach, the upstream educed resistance is greater than the downstream educed resistance, while the opposite is true for the $0 \mathrm{D}$ eduction approach at low frequencies. The same observation applies to the reactance at all frequencies.

\subsection{Discussion}

Overall, the impedance mismatch can be significantly reduced (near the antiresonance for the CT57 and at high frequencies for the GE03), when compared to the classical indirect eduction method. For the CT57, most of the remaining mismatch is at lower frequencies and near the antiresonance. This, however, could be partly explained by the uncertainties of the wavenumber measurements at low frequencies (due perhaps to the ratio of liner length over wave length becoming small), and by an increased uncertainty at antiresonance where the liner is nearly non absorbent. For 


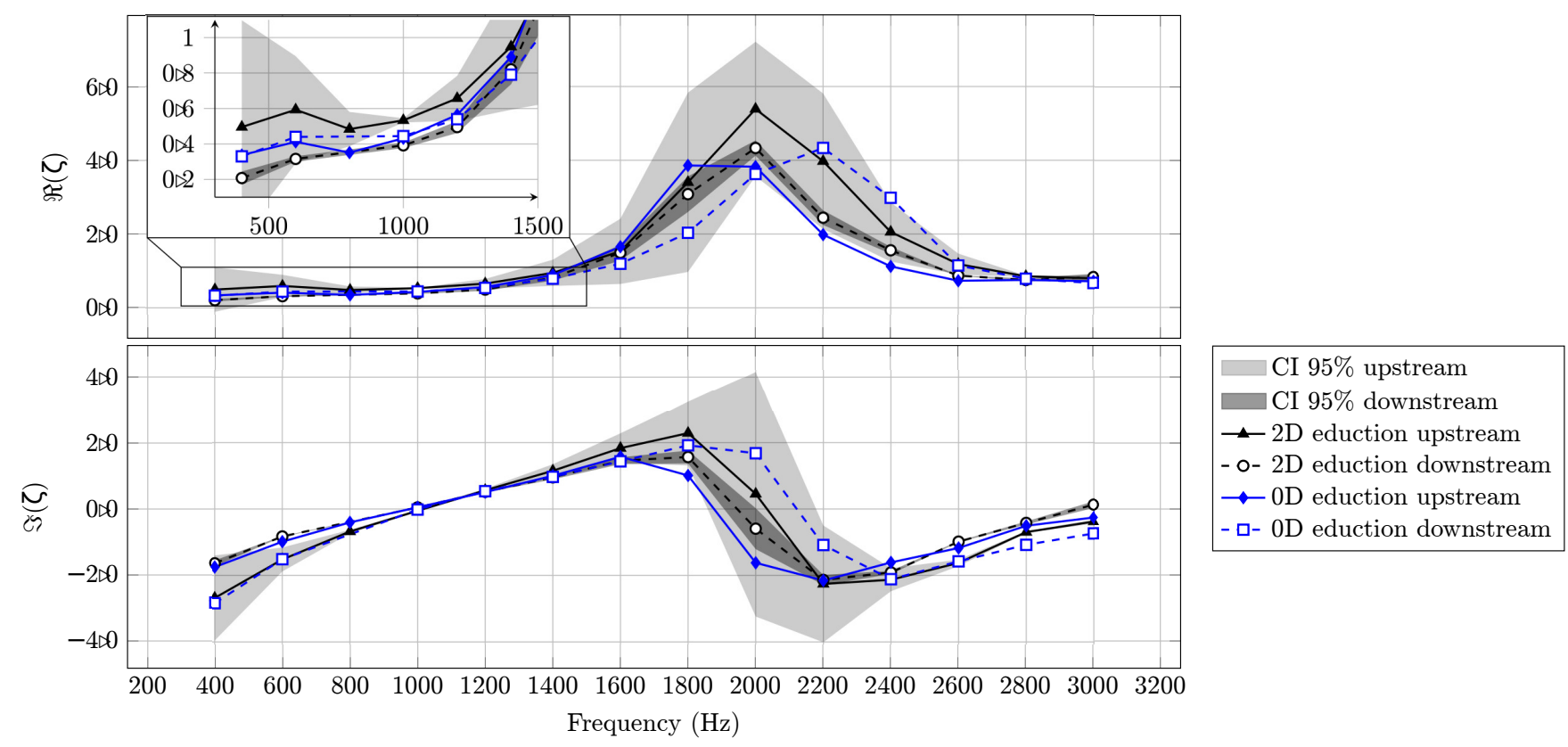

Figure 11: Educed impedance for the CT57 using the 2D eduction of Eq. 19 and the classical 0D eduction Eq. 2. Credibility intervals are displayed on the 2D eduction results.

the GE03, the mismatch amplitude remains identical at low frequencies, when compared to the straightforward approach, but of opposite sign.

The uncertainties that were obtained on the educed impedance via the Bayesian inference strategy of Sec. 4.1 seem to correlate well with the amplitude of the impedance mismatch, which is defined for the resistance as

$$
\Delta_{ \pm}(\Re(\zeta))=\left|\Re\left(\zeta^{+}\right)-\Re\left(\zeta^{-}\right)\right|
$$

with a similar definition for the reactance mismatch. To back-up the above claim, the educed values of $\sigma_{\Re(\zeta)}$ (respectively $\left.\sigma_{\Im(\zeta)}\right)$ are plotted against the associated $\Delta_{ \pm}(\Re(\zeta))$ (respectively $\Delta_{ \pm}(\Im(\zeta))$ ) in Fig. 13 for both liner samples. Notice the presence of two distinct ordinate scales, due to the high difference in values between upstream $\left(\sigma_{\zeta}^{+}\right)$and downstream $\left(\sigma_{\zeta}^{-}\right)$standard deviations. For the CT57, a clustering of the points near the origin is observed when plotting the real part of the impedance standard deviation $\sigma_{\Re(\zeta)}$ against the real part of the impedance mismatch. However, things are not as clear cut when looking at the reactance. For the GE03 material, however, a strong positive correlation appears between uncertainties and impedance mismatch, as seen by the straight lines of positive slopes. These correlations indicate that the phenomenon responsible for the impedance mismatch (leading to high $\Delta_{ \pm}(\zeta)$ values) might be similar in nature to the phenomenon responsible for the eduction uncertainties (shown by $\sigma_{\zeta}^{ \pm}$). Yet, since the source of uncertainties is fairly well explained by the poor liner efficiency in some frequency bands, or by the difficulty in evaluating the wavenumber when the frequency is too low, it stands to reason that the impedance mismatch that has been observed so far in the literature can be at least partly explained by a combination of a wrong definition of the wavenumber, and a high uncertainty in the eduction.

A side comment is that the problem of a biased wavenumber definition is irrelevant for axisymmetric ducts, where only a 1D flow profile information would be sufficient. In cylindrical 


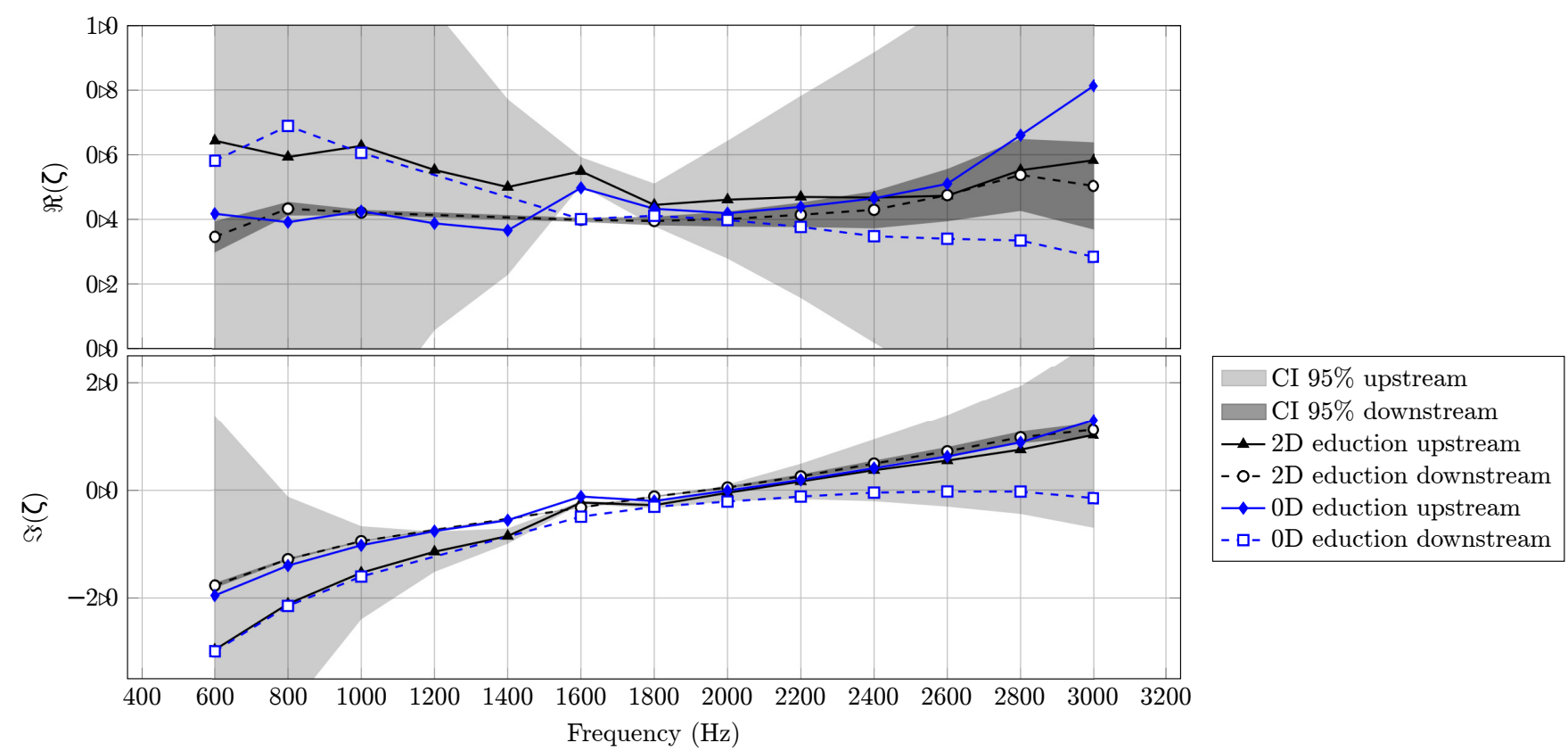

Figure 12: Educed impedance for the GE03 using the 2D eduction of Eq. 19 and the classical 0D eduction Eq. 2 .

ducts, however, it becomes more difficult to assess the wavenumber of the waves propagating in the lined section, because it is experimentally tedious, if not impossible, to insert a microphone or any measurement probe within the lined part of the test rig. Other eduction methods remain possible [38, 39], using only upstream and downstream measurements. In practice, creating liners to cover circular ducts can prove problematic and require multiple separated elements, due to the high curvature. The introduction of splices between these elements would result in a biased wavenumber evaluation, adding to the problem complexity [40]. Another way to eventually bypass the bias in wavenumber definition would be to consider direct in-situ impedance measurements [41].

\section{Conclusions}

A significant amount of eduction procedures in the literature have focused on the use of direct relationships between wavenumber and impedance, or simplified 2D numerical schemes, and/or an Ingard-Myers boundary condition at the impedance location. However, there is an apparent mismatch between wavenumber definitions when considering these approximations, as made explicit in Sec. 2.2.

This study has shown that the mismatch in educed impedance, when considering downstream or upstream source locations, could partially be explained by the wavenumber mismatch. When performing eductions based on a 1D calculation of the propagation wavenumber (infinite liner hypothesis), the influence of the transverse (i.e., spanwise direction) flow profile is not taken into account, which biases the eduction. The same problem arises when considering a longitudinal 2D LEE/CHE numerical scheme, where the transverse direction is ignored, as is currently the case in most publications on the subject. It is thus advised that future eduction studies characterize the flow profile in the full cross-section, instead of using only a midspan measurement. Further, viscous effects should be accounted for using the linearized Navier-Stokes equations instead of the LEE, as was done for instance in Refs. [26, 28. While the influence of viscous effects has been shown 


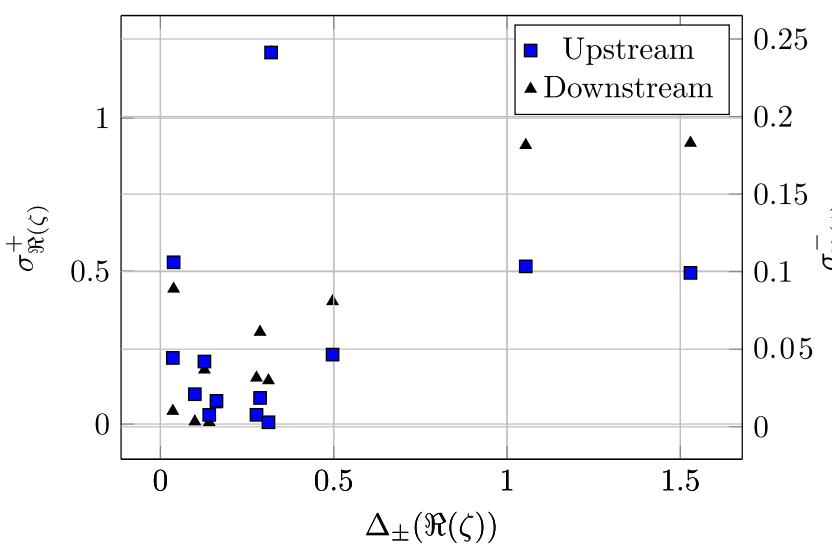

(a) CT57 : real part.

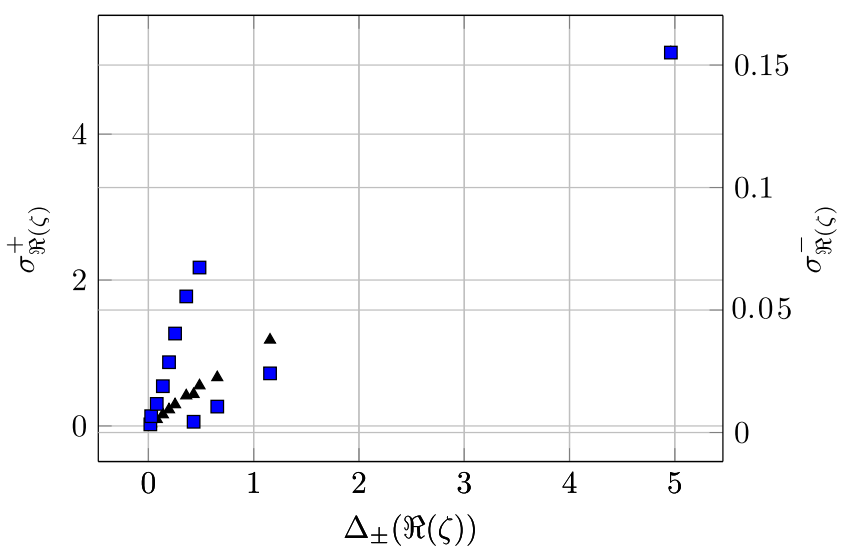

(c) GE03 : real part.

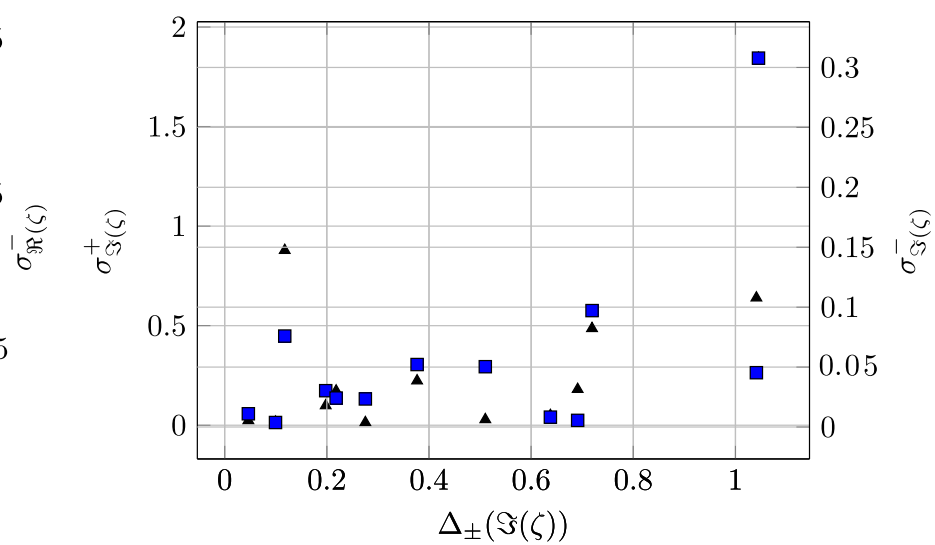

(b) CT57 : imaginary part.

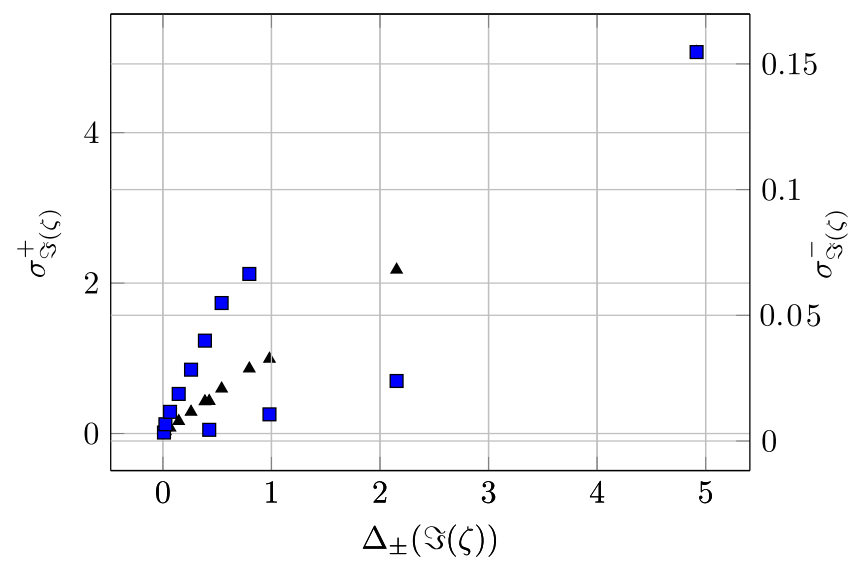

(d) GE03 : imaginary part.

Figure 13: Evolution of the uncertainty as a function of the impedance mismatch.

to not be solely responsible for the impedance mismatch [26], their effect is sufficiently strong to bias eductions not accounting for them.

An updated eduction was proposed, that uses a 2D resolution of the LEE in the cross-section of the aeroacoustic bench to compute a numerical wavenumber that is compared to a measured one. A statistical inference process based on Bayes rule is used to account for the presence of experimental and modeling uncertainties. This eduction process shows a reduced impedance mismatch for the two liners considered in a certain frequency range. It is also shown that a correlation exists between the remaining impedance mismatch and the uncertainties obtained via the Bayesian inference eduction, showing that the two quantities might relate to the same phenomenon (larger uncertainties when the liner is not absorbent). Another possibility is the presence of unmodeled physics of the impedance boundary condition, which is still an ongoing research topic [42, 43]. However, the present work shows that the refinement of physics modeling will require accounting for the experimental uncertainties in impedance eduction.

Future attempts at improving the impedance models should thus account for uncertainties and for the wavenumber bias introduced during the eduction process. 


\section{Acknowledgments}

This work is part of the NASA/ONERA collaborative agreement on aircraft noise reduction.

\section{References}

[1] U. Ingård, S. Labate, Acoustic circulation effects and the nonlinear impedance of orifices, The Journal of the Acoustical Society of America 22 (2) (1950) 211-218. doi:10.1121/1.1906591.

[2] T. H. Melling, The acoustic impendance of perforates at medium and high sound pressure levels, J. Sound Vib. 29 (1) (1973) 1-65. doi:10.1016/S0022-460X(73)80125-7.

[3] M. Howe, On the theory of unsteady high reynolds number flow through a circular aperture, Proc. R. Soc. Lond. A 366 (1725) (1979) 205-223. doi:10.1098/rspa.1979.0048.

[4] A. Cummings, W. Eversman, High amplitude acoustic transmission through duct terminations: Theory, Journal of Sound and Vibration 91 (4) (1983) 503-518. doi:10.1016/ 0022-460X (83)90829-5.

[5] X. Jing, X. Sun, Sound-excited flow and acoustic nonlinearity at an orifice, Physics of Fluids 14 (1) (2002) 268-276. doi:10.1063/1.1423934.

[6] A. Guess, Calculation of perforated plate liner parameters from specified acoustic resistance and reactance, J. Sound Vib. 40 (1) (1975) 119-137. doi:10.1016/S0022-460X (75)80234-3.

[7] A. Goldman, R. L. Panton, Measurement of the acoustic impedance of an orifice under a turbulent boundary layer, The Journal of the Acoustical Society of America 60 (6) (1976) 1397-1405. doi:10.1121/1.381233.

[8] A. Cummings, The effects of grazing turbulent pipe-flow on the impedance of an orifice, Acta Acustica United with Acustica 61 (4) (1986) 233-242.

[9] R. Kirby, A. Cummings, The impedance of perforated plates subjected to grazing gas flow and backed by porous media, J. Sound Vib. 217 (4) (1998) 619-636. doi:10.1006/jsvi. 1998.1811.

[10] J. M. Roche, Simulation numérique de l'absorption acoustique de matériaux résonants en présence d'écoulement, Ph.D. thesis, Université du Maine (2011).

[11] Q. Zhang, D. J. Bodony, Numerical investigation of a honeycomb liner grazed by laminar and turbulent boundary layers, J. Fluid Mech. 792 (2016) 936-980. doi:10.1017/jfm.2016.79.

[12] X. Sun, X. Jing, H. Zhang, Y. Shi, Effect of grazing-bias flow interaction on acoustic impedance of perforated plates, J. Sound Vib. 254 (3) (2002) 557-573. doi:10.1006/jsvi. 2001.4110 .

[13] Q. Zhang, Direct numerical investigation and reduced-order modeling of 3-d honeycomb acoustic liners, Ph.D. thesis, University of Illinois at Urbana-Champaign (2014). URL http://hdl .handle.net/2142/49603 
[14] X. Jing, S. Peng, X. Sun, A straightforward method for wall impedance eduction in a flow duct, The Journal of the Acoustical Society of America 124 (1) (2008) 227-234. doi:10. $1121 / 1.2932256$.

[15] T. Elnady, H. Bodén, B. Elhadidi, Validation of an inverse semi-analytical technique to educe liner impedance, AIAA journal 47 (12) (2009) 2836-2844. doi:10.2514/1.41647.

[16] L. Zhou, H. Boden, C. Lahiri, F. Bake, L. Enghardt, S. Busse-Gerstengarbe, T. Elnady, Comparison of impedance eduction results using different methods and test rigs, in: 20th AIAA/CEAS Aeroacoustics Conference, 2014, p. 2955. doi:10.2514/6.2014-2955.

[17] U. Ingard, Influence of fluid motion past a plane boundary on sound reflection, absorption, and transmission, The Journal of the Acoustical Society of America 31 (7) (1959) 1035-1036. doi:10.1121/1.1907805.

[18] M. Myers, On the acoustic boundary condition in the presence of flow, Journal of Sound and Vibration 71 (3) (1980) 429-434. doi:10.1016/0022-460X (80)90424-1.

[19] M. G. Jones, W. R. Watson, T. L. Parrott, Benchmark data for evaluation of aeroacoustic propagation codes with grazing flow, AIAA paper 2853 (2005) 2005. doi:10.2514/6. 2005-2853.

[20] W. Eversman, J. M. Gallman, Impedance eduction with an extended search procedure, AIAA journal 49 (9) (2011) 1960-1970. doi:10.2514/1.J050831.

[21] J. Primus, E. Piot, F. Simon, An adjoint-based method for liner impedance eduction: Validation and numerical investigation, J. Sound Vib. 332 (1) (2013) 58-75. doi:10.1016/j.jsv. 2012.07 .051 .

[22] R. Roncen, F. Méry, E. Piot, F. Simon, Statistical inference method for liner impedance eduction with a shear grazing flow, AIAA Journal (2018) 1-11doi:10.2514/1.J057559.

[23] Y. Renou, Y. Aurégan, Failure of the ingard-myers boundary condition for a lined duct: An experimental investigation, The Journal of the Acoustical Society of America 130 (1) (2011) 52-60. doi:10.1121/1.3586789.

[24] A. Spillere, J. A. Cordioli, H. Bodén, On the effect of boundary conditions on impedance eduction results, in: 23rd AIAA/CEAS Aeroacoustics Conference, 2017, p. 3185. doi:10. $2514 / 6.2017-3185$

[25] H. Bodén, J. A. Cordioli, A. Spillere, P. Serrano, Comparison of the effect of flow direction on liner impedance using different measurement methods, in: 23rd AIAA/CEAS Aeroacoustics Conference, 2017, p. 3184. doi:10.2514/6.2017-3184.

[26] C. Weng, A. Schulz, D. Ronneberger, L. Enghardt, F. Bake, Flow and viscous effects on impedance eduction, AIAA Journal 56 (3) (2017) 1118-1132. doi:10.2514/1. J055838.

[27] W. R. Watson, M. G. Jones, A comparative study of four impedance eduction methodologies using several test liners, in: 19th AIAA/CEAS Aeroacoustics Conference, 2013, p. 2274. doi:10.2514/6.2013-2274. 
[28] C. Weng, A. Schulz, D. Ronneberger, L. Enghardt, F. Bake, Impedance eduction in the presence of turbulent shear flow using the linearized navier-stokes equations, in: 23rd AIAA/CEAS Aeroacoustics Conference, 2017, p. 3182. doi:10.2514/6.2017-3182.

[29] D. M. Nark, M. G. Jones, E. Piot, Assessment of axial wave number and mean flow uncertainty on acoustic liner impedance eduction, in: 2018 AIAA/CEAS Aeroacoustics Conference, 2018, p. 3444. doi:10.2514/6.2018-3444.

[30] W. R. Watson, M. G. Jones, Impedance eduction in a duct using linearized euler equations, in: 2018 AIAA/CEAS Aeroacoustics Conference, 2018, p. 3442. doi:10.2514/6.2018-3442.

[31] R. Roncen, E. Piot, F. Mery, F. Simon, M. G. Jones, D. M. Nark, Influence of source propagation direction and shear flow profile in impedance eduction of acoustic liners, in: 25th AIAA/CEAS Aeroacoustics Conference, 2019, p. 2469. doi:10.2514/6.2019-2469.

[32] R. Kumaresan, D. Tufts, Estimating the parameters of exponentially damped sinusoids and pole-zero modeling in noise, IEEE Transactions on Acoustics, Speech, and Signal Processing 30 (6) (1982) 833-840. doi:10.1109/TASSP .1982.1163974.

[33] L. Pascal, E. Piot, G. Casalis, Discontinuous Galerkin method for the computation of acoustic modes in lined flow ducts with rigid splices, Journal of Sound and Vibration 332 (13) (2013) 3270 - 3288. doi:10.1016/j.jsv.2013.01.021.

[34] G. Boyer, E. Piot, J.-P. Brazier, Theoretical investigation of hydrodynamic surface mode in a lined duct with sheared flow and comparison with experiment, Journal of Sound and Vibration 330 (8) (2011) 1793-1809. doi:10.1016/j.jsv.2010.10.035.

[35] S. Rienstra, G. Vilenski, Spatial instability of boundary layer along impedance wall, in: 14th AIAA/CEAS Aeroacoustics Conference (29th AIAA Aeroacoustics Conference), 2008, p. 2932. doi:10.2514/6.2008-2932.

[36] R. C. Smith, Uncertainty quantification: theory, implementation, and applications, Vol. 12, Siam, Philadelphia, 2013.

[37] Y. Buot de l'Epine, J.-D. Chazot, J.-M. Ville, Acoustical impedance characterization of liners using a bayesian approach, in: Proceedings of Meetings on Acoustics ICA2013, Vol. 19, ASA, 2013, p. 030108.

[38] Y. Aurégan, M. Leroux, V. Pagneux, Measurement of liner impedance with flow by an inverse method, in: 10th AIAA/CEAS Aeroacoustics Conference, 2004, p. 2838.

[39] M. Taktak, J. M. Ville, M. Haddar, G. Gabard, F. Foucart, An indirect method for the characterization of locally reacting liners, The Journal of the Acoustical Society of America 127 (6) (2010) 3548-3559. doi:10.1121/1.3365250.

[40] L. Pascal, E. Piot, G. Casalis, Discontinuous galerkin method for the computation of acoustic modes in lined flow ducts with rigid splices, Journal of Sound and Vibration 332 (13) (2013) 3270-3288. doi:10.1016/j.jsv.2013.01.021.

[41] P. Dean, An in situ method of wall acoustic impedance measurement in flow ducts, Journal of Sound and Vibration 34 (1) (1974) 97-IN6. 
[42] A. Schulz, C. Weng, F. Bake, L. Enghardt, D. Ronneberger, Modeling of liner impedance with grazing shear flow using a new momentum transfer boundary condition, in: 23rd AIAA/CEAS Aeroacoustics Conference, 2017, p. 3377. doi:10.2514/6.2017-3377.

[43] Y. Aurégan, On the use of a stress-impedance model to describe sound propagation in a lined duct with grazing flow, The Journal of the Acoustical Society of America 143 (5) (2018) 2975-2979. doi:10.1121/1.5037585. 